\title{
Damage Detection in Composites By Artificial Neural Networks Trained By Using in Situ Distributed Strains
}

\section{America Califano ${ }^{1,2}$ D Neha Chandarana ${ }^{2,3}$ • Luigi Grassia ${ }^{1}$ • Alberto D'Amore ${ }^{1}$ • Constantinos Soutis ${ }^{2,4}$}

Received: 26 June 2020 / Revised: 26 June 2020 / Accepted: 21 July 2020 /Published online: 7 August 2020

(C) The Author(s) 2020

\begin{abstract}
In this paper, a passive structural health monitoring (SHM) method capable of detecting the presence of damage in carbon fibre/epoxy composite plates is developed. The method requires the measurement of strains from the considered structure, which are used to set up, train, and test artificial neural networks (ANNs). At the end of the training phase, the networks find correlations between the given strains, which represent the 'fingerprint' of the structure under investigation. Changes in the distribution of these strains is captured by assessing differences in the previously identified strain correlations. If any cause generates damage that alters the strain distribution, this is considered as a reason for further detailed structural inspection. The novelty of the strain algorithm comes from its independence from both the choice of material and the loading condition. It does not require the prior knowledge of material properties based on stress-strain relationships and, as the strain correlations represent the structure and its mechanical behaviour, they are valid for the full range of operating loads. An implementation of such approach is herein presented based on the usage of a distributed optical fibre sensor that allows to obtain strain measurement with an incredibly high resolution.
\end{abstract}

Keywords Structural health monitoring · Composite materials · Machine learning · Optical fibres · Distributed strain sensing

America Califano

america.califano@unicampania.it; america.califano@gmail.com

Constantinos Soutis

constantinos.soutis@manchester.ac.uk

1 Department of Engineering, University of Campania "Luigi Vanvitelli", Aversa 81031, Italy

2 i-Composites Lab, The University of Manchester, Manchester, UK

3 Henry Royce Institute, The University of Manchester, Manchester, UK

4 Aerospace Research Institute, The University of Manchester, Manchester, UK 


\section{Introduction}

Fibre reinforced composite materials exhibit superior mechanical properties, in terms of specific strength and modulus, compared to metallic materials. They are desirable for use in the aeronautical and aerospace industries due to the increasing demand for lightweight structures. The content of composites in aircraft structures has increased from less than 5\% in the late $80 \mathrm{~s}$ to more than $50 \%$ in recent years [1]. The design of composite structures is often complex due to the challenges associated with their manufacture and the effect on the resulting mechanical properties. The manufacturing process may introduce defects such as voids, resin rich regions, and misalignment of fibres. The design of composite structures becomes more critical where the concerned structures are subjected to anomalous or cyclic loading conditions when in service [2, 3]. In addition, the wide range of damage and defects that can occur independently and simultaneously has made it fundamental to monitor structures in real-time to detect anomalies at an early stage. Furthermore, as aircraft travel is increasing considerably, the research community is driven towards the simplified application of structural inspection methods, leading to quicker maintenance procedures.

At present, for the assessment of the safety, structural integrity, and durability of engineering structures, non-destructive evaluation (NDE) methods are widely used. The most common techniques include visual inspection, eddy-current, thermography, optical interferometry, ultrasonic inspection, radiography, and vibration/modal analysis [4]. Assessments are conducted at regularly scheduled intervals, but real-time structural health information is obtained. For this reason, permanently integrated structural health monitoring (SHM) techniques have recently gained more interest. SHM techniques aim to provide real-time diagnoses of the host structure, at the local and global scale [4]. An SHM system will generally involve the permanent integration of sensors (e.g. strain gauges, piezoelectric transducers, and optical fibres), transmission of the recorded data, and computational processing of the data to form a diagnosis. SHM techniques can be classified as active or passive. Active methods infer the health of the structure by exciting it in some way, at defined regular intervals; assessments are performed on-demand. Passive ones continuously acquire data from the structure without providing any excitation; the data is a direct result of changes in the structure that occur due to the onset of damage. Typical examples of active and passive SHM techniques include the use of guided waves [5-7] and the evaluation of static-parameters (e.g. strains and displacements), respectively [8]. Strain-based approaches are common and tend to make use of electrical strain gauges that are adhesively bonded to the structure. In the case of composites, the use of distributed optical fibre sensors (DOFS) is appealing due to their light weight and small size. Optical fibres have been used successfully for quasi-distributed [7] and distributed strain monitoring [9]. In addition, fibre optic sensors are particularly suitable for the health monitoring of large structures as well, because they provide distributed sensing over long distances. The small size of fibre optic sensors ( $<250$ micron in diameter) imposes negligible intrusion into the host structure and allows fast interrogation with minimal wiring requirements [10].

As the loss of structural integrity mainly relies on the failure of materials resulting from deformation and strain concentrations, the analysis of strain fields seems to be useful and likely to lead to more reliable predictions aimed to reduce premature or unnecessary repairs. The product changes are usually classified into 'minor' or 'major', depending on the entity of the effect on balance, structural reliability, weight, and other operational characteristics. Minor repairs are generally correlated to the so-called 'Category 1 Damage' that refers to barely visible impact damage (BVID) and to all those damage configurations that have proved to be 
sustained by the in-service structures [11]. Such category is related to the use of NDE in service applications and it has been recently supported by appropriate SHM tools, in order to establish cheap, reliable and robust procedures.

\subsection{Machine Learning as a Tool for Structural Health Monitoring}

The data acquired from SHM systems (both active and passive) may be considered as belonging to the "big data" type, due to their wide complexity, variability, and diversity. Many challenges may be encountered when dealing with big data, such as difficulty in searching, capturing, and storing significant data, as well as in building adequate computational architectures and data-processing models. So, the appeal of the big-data field has led to the development of sophisticated algorithms and computing platforms that make it possible to reduce the size of the data itself [12].

Damage is defined as something that causes a modification to a material and/or its geometry, which leads to a change in its mechanical performance. Machine learning based SHM in the context of composite materials requires the definition of indicators that relate to different damage, based on the evaluation of measured data and features. Machine learning approaches can be described as either supervised or unsupervised. Supervised and partially supervised approaches require prior label information, i.e. there is a set of training data available, where the relationship between the measured data and physical damage phenomena is known. This prior knowledge is then applied when dealing with the analysis of new datasets. Unsupervised approaches do not make use of prior information and can be considered as 'blind'. They make use of patterns and similarities in data features in order to find labels [13].

Though unsupervised approaches can be more adaptable, supervised and partially-supervised methods are often preferred in applications where the signature of damage is well characterised and understood. Algorithms based on artificial neural networks (ANNs) have been developed for damage detection in wind turbine blades [14]. The use of auto-associative ANNs in this application has enabled a smaller subset of sensors to be used for SHM of the whole turbine blade, therefore significantly reducing the size of the dataset. Convolutional neural networks have also been shown to be successful in civil engineering applications [15] and in fatigue analysis of aircraft structures combined with Lamb waves [16]. Other examples may be found in $[17,18]$. In both cases, the damage identification on aerospace composite structures has been carried out through Machine Learning tools (such as a self-organising map for pattern recognition and ANNs based on dynamic measurements). Finally, a model for the damage detection in composite structures based on feedforward neural networks trained via strain data measured on real test cases may be found in [8]; the latter will be employed in the following sections.

According to literature, machine learning algorithms seem to be very useful tools for SHM purposes as they are able to automatically extract patterns in groups of data after being properly trained. In particular, ANNs are desirable when dealing with damage identification problems that may be classified as typical examples of binary learning ('healthy' or 'damaged'). A neural network able to implement binary learning can be modelled following two approaches: discrimination-based learning or recognition-based learning. In the first approach, the network is trained using both 'positive' and 'negative' samples in order to learn how to discriminate among them; in the second, the network is trained using only 'positive' samples and it is able to recognise only these [19]. Damage diagnosis represents a typical engineering problem for which it is almost impossible to forecast all the 'negative' events, since this would mean to be able to discover all the possible perturbations that a structural component would suffer as damage. 


\subsection{Strain-based Structural Health Monitoring Methods}

Static-parameter-based techniques are locally sensitive to defects, simple to operate, and costeffective to implement. They rely on the assumption that the presence of damage causes changes in the distribution of displacements and strains compared with a 'pristine' state. The strain of a structure under load can be readily measured and monitored over time using electrical strain gauges or optical fibre-based sensors, which can be placed in known structural 'hotspots', where defects or damage under certain loading conditions could trigger premature failure.

When dealing with strain data, some challenges are often encountered. As the size of collected data is generally extended, it is necessary to perform unfolding, centring and scaling data operations before any damage assessment [20]. These operations are generally combined with principal component analysis (PCA) that allows to extrapolate the main features of data sets in order to obtain a new set of input data that is the linear combination of the original ones [21]. This is useful to reduce the dimensionality of data-rich strain fields and, consequently, to limit their redundancy. Nevertheless, as the data-reduction operations usually involve the whole set of data, in real-life applications, they might be undesirably time-consuming and computationally demanding. In addition, most of the methods presented in open literature:

(i) rely on the combination of strain measurements with other techniques (such as acoustic emission and digital image correlation) [22];

(ii) they are strictly dependent on the evaluation of the material's stress-strain behaviour [23];

(iii) they are unavoidably related to the evaluation of a high number of damage patterns (e.g. cracks of different lengths and entities) [24].

Finally, although these models and methods have shown their reliability in providing damage assessment in different scenarios, it remains a challenge to obtain information on damage occurrence just from strain measurement and mapping [25] because even high strain values would not necessarily indicate the presence of damage. This is essentially due to the highly scattered mechanical properties of multi-layered composites [26] and the variability and unpredictability of internal damage modes, especially when they interact [27].

The procedure firstly presented in [8] and herein discussed aims to go over the previous aspects, introducing some novelties in the strain-based methods field.

\subsection{Contribution}

As a contribution to the development of new ways to process data collected from sensors, in this work a method for damage detection is studied. The method has been presented for the first time in [8], where Grassia et al.. proposed their damage diagnosis strain algorithm for composites' Structural Health Monitoring and they proved it against two experimental aeronautical test cases equipped with networks of strain-gauges.

The proposed methodology relates strains measured in some locations across a structure to the strains measured in their 'neighbourhood', based on the idea that strains in neighbouring locations are representative of the deformations in those locations. So, defining correlations among those strains means defining a law that characterises the evaluated structure's mechanics, even if its mechanical properties are not known. The correlations among strains are assessed through one-layer feed-forward neural networks that are trained with strain data collected on the pristine configuration of the evaluated structure. The novelty of the method 
stands in its independence from both the choice of material and the loading condition. The method does not require the prior knowledge of material properties based on stress-strain relationships and, as the strain correlations represent the structure and its mechanical behaviour, they are valid for the full range of operating loads. In the framework of the proposed approach there are no damage patterns to correlate to strains, so it is not necessary to perform data reduction or classification operations, in contrast with the methods available in literature. This is essentially due to the exceptional extrapolation and recognition capabilities of ANNs [28].

The current work shows an implementation of the aforementioned method on in a novel experimental scenario. The test case is a carbon fibre reinforced polymer (CFRP) plate comprising an embedded DOFS. The specimen is subjected to four-point bending tests during which strain data are collected by the DOFS and, then, analysed through the help of one-layer feed-forward neural network trained via back-propagation algorithm. The novelty stands in testing the experimental case using a distributed sensor and in assessing the potentiality of both ANNs and the method when handling large-dimensions data sets, as the ones collected by the FOS.

\section{ANN-based Damage Diagnosis Procedure}

The methodology applied for data analysis is described below, while for further details on the algorithm the reader is referred to [8].

The present diagnosis procedure uses transfer functions that correlate the strains experienced in neighbouring locations on the host structure, independently of the externally applied loads. Strains are recorded in discrete locations on the host structure. When relative strain comparisons are made, these discrete locations are termed 'master' and 'slave' nodes. The correlations between the strains at master and slave nodes act as a 'fingerprint' of the structure and are established using ANNs. Each master-slave location pair has its own neural network, which describes the relationship between strains in their respective locations. Once the networks have been initialised, the algorithm is ready to be used. The logic on which the procedure is based relies on the assumption that only the locations in the 'neighbourhood' (i.e. in close proximity) of a damage event are affected by its presence, while locations that are sufficiently far from the damage are not sensitive to its presence. Figure 1 describes the steps of the algorithm applied to the recorded strain data. A step-by-step description of the procedure is given below:

Step 1. First, the reference structure is subjected to operational loads - in this case, $0.55 \mathrm{kN}$. During loading, the strains in both master and slave locations $\left(\left\{\epsilon_{m, s}^{R}\right\}\right)$ are recorded.

Step 2. Then introducing some damage on the structure and re-subjecting it to the operational loads, the strains $\left(\left\{\epsilon_{m, s}^{D}\right\}\right)$ are measured again.

Step 3. After the acquisition of all the necessary data, the core of the procedure is implemented. The strains measured on the reference structure are used to train the neural networks. The strains measured in slave locations $\left(\left\{\epsilon_{s}^{R}\right\}\right)$ are used as input data, while those measured in master locations $\left(\left\{\epsilon_{m}^{R}\right\}\right)$ are used as output data. 


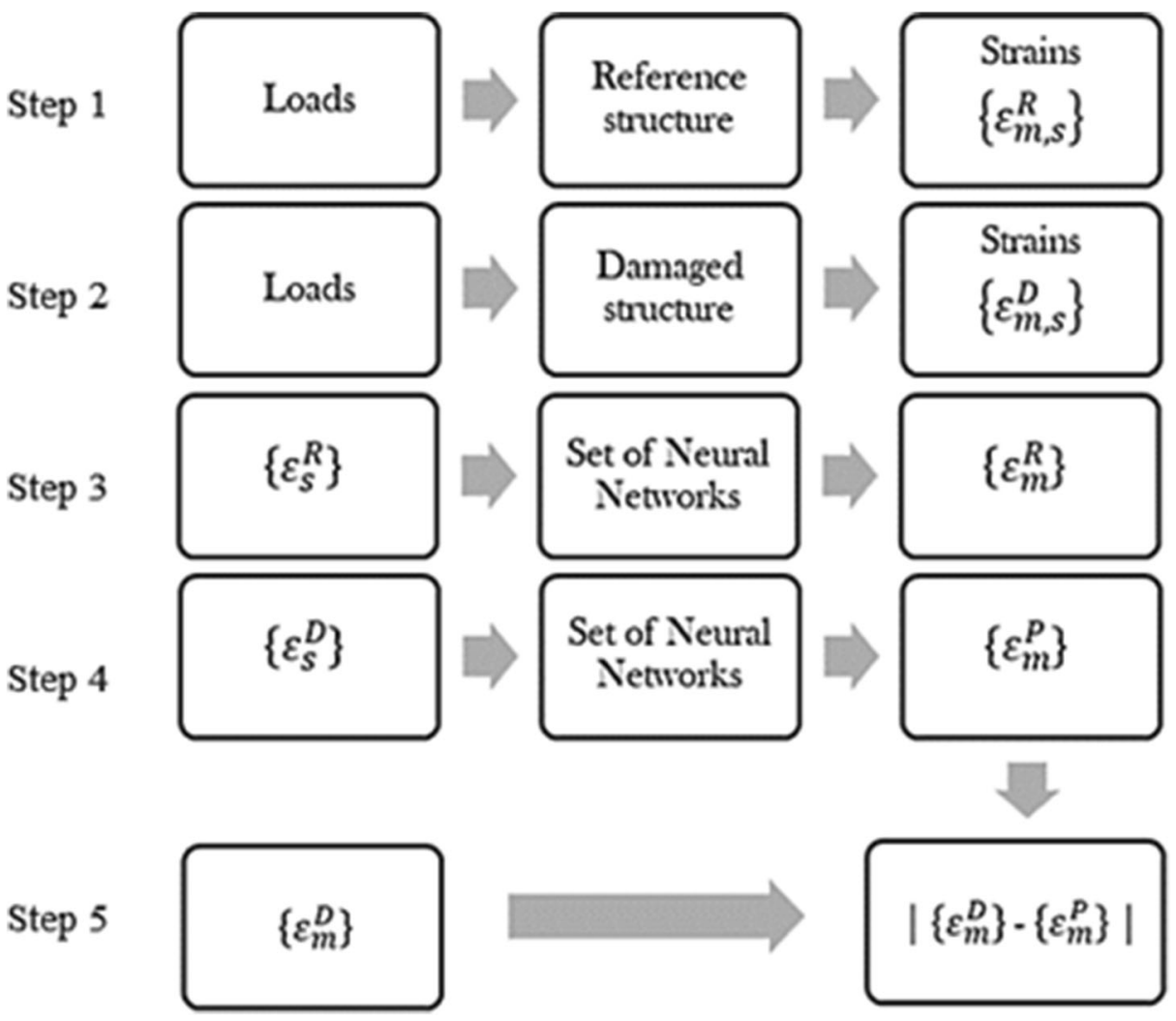

Fig. 1 Damage diagnosis procedure flowchart

Step 4. At this point, the strains measured in the slave locations on the damaged structure ( $\left\{\epsilon_{s}^{D}\right\}$ ) are provided as new input data to the trained networks. The networks produce the strain predictions at master locations $\left(\left\{\epsilon_{m}^{P}\right\}\right)$ as output data.

Step 5. The strain predictions output by Step 4 are compared to the actual strains measured on the damaged structure $\left(\left\{\epsilon_{m}^{D}\right\}\right)$. The evaluation of the mismatch $(e)$, shown in Eq. (1), leads to the detection of damage:

$$
e=\left|\left\{\epsilon_{m}^{D}\right\}-\left\{\epsilon_{m}^{P}\right\}\right|
$$

As the ANNs have been trained using strains from the reference structure, if new input data not belonging to the training set (e.g. strain measured near damaged area) are provided, they will respond with 'out-of-scale' output data. This means that, in this case, ANNs are not able to provide reliable predictions $\left(\left\{\epsilon_{m}^{P}\right\}\right)$ of the experimentally measured data $\left(\left\{\epsilon_{m}^{D}\right\}\right)$ and, so, the mismatch reported in Eq. (1) shows non-negligible values. This happens because, as the local structural stiffness has changed due to damage, the correlations between $\left\{\epsilon_{s}^{R}\right\}$ and $\left\{\epsilon_{m}^{R}\right\}$, found during the training phase and established as transfer function of ANNs, will not apply anymore. The correlation between strains directly relates to the mechanical features of the analysed structure: the deformation 
capability of a structure depends on its stiffness. So, as soon as some generic cause leads to the formation of damage, the mechanical features of structure itself (such as stiffness) change, and so do both its deformation and the strain correlations.

It has to be mentioned that during the neural networks training phase (Step 2), as there is no universal technique to choose the number of hidden neurons, the number of neurons for each network has been chosen adopting a simple procedure:

1 Training each network several times increasing the number of hidden neurons $k$;

2 Evaluating the Performance Index of each training, defined in Eq. (2):

$$
P_{n, L_{R_{t}}}^{k}=\sqrt{\frac{1}{L_{R_{t}}} \sum_{l=1}^{L_{R_{t}}}\left(\epsilon_{n, l}^{R P}-\epsilon_{n, l}^{R}\right)^{2}}
$$

where $\epsilon_{n . l}^{R P}$ represents the strain predicted by the $n$-th network for the reference structure under the $l$-th loading step, $\epsilon_{n, l}^{R}$ represents the strain measured on the reference structure under the $l$-th loading step, $L_{R}$ is the total number of loading steps applied on the reference structure and $L_{R_{t}}$ is the number of load steps picked randomly from the database, with 1determining the optimum hidden neurons number $\left(k_{n}\right)$ as shown in Eq. (3):

$$
\forall n \leq N, k_{n}=k \mid P_{n, L_{R_{t}}}=\inf \left\{\mathrm{P}_{\mathrm{n}, \mathrm{L}_{\mathrm{R}_{\mathrm{t}}}}^{\mathrm{k}}\right\}_{\mathrm{k}=1, \mathrm{H}}, \mathrm{k}=1,, \mathrm{H}, \mathrm{H} \in \mathbb{N}
$$

where $\mathrm{N}$ is the total number of ANNs and $\mathrm{H}$ is the maximum number of hidden neurons used.

\section{Implementation of the Proposed ANN on an Experimental Case}

The described damage diagnosis procedure is implemented on strain data measured from a fibre reinforced polymer composite plate $(400 \mathrm{~mm}$ x $200 \mathrm{~mm})$ that consists of eight plies of carbon fibre fabric (PX35-13 $50 \mathrm{k}$ unidirectional fabric, supplied by Zoltek) infused with an epoxy-based resin system (Araldite LY564 and Aradur 2594, supplied by Huntsman, Basel, Switzerland).

The plate was a cross-ply symmetric laminate, with orientation $\left[90 / 0 / 0_{\mathrm{OFS}} / 90\right]_{\mathrm{S}}$, in which, during the lay-up process, a single-mode, polyimide-coated silica glass DOFS (of $155 \mu \mathrm{m}$ diameter and $2 \mathrm{~m}$ length) was embedded (in a 0 -degree layer, named $0_{\mathrm{OFS}}$ ), so that it could be parallel to the fibre direction (Fig. 2). The plate was manufactured by vacuum assisted resin infusion moulding (VARIM) [29] followed by an oven curing process, as detailed in [7]. During composite fabrication, the development of strains was monitored in situ and in real time.

After the manufacturing process, several quasi-static four-point bending tests were conducted on the plate, following the ASTM D7264 standard [30] on an Instron 5969 universal testing machine fitted with a $50 \mathrm{kN}$ load cell. To ensure uniform distribution of the load across the width of the panel, aluminium 'spreader bars' were placed between the loading noses and the specimen.

The first loading cycle was conducted on the plate in the pristine condition, reaching a maximum applied load of $0.55 \mathrm{kN}$. Then, a $2.5 \mathrm{~mm}$ diameter hole was drilled in the centre of the plate and this new configuration was subjected to further four-point bending, reaching again a maximum applied load of $0.55 \mathrm{kN}$. Between successive cycles of loading, the diameter 


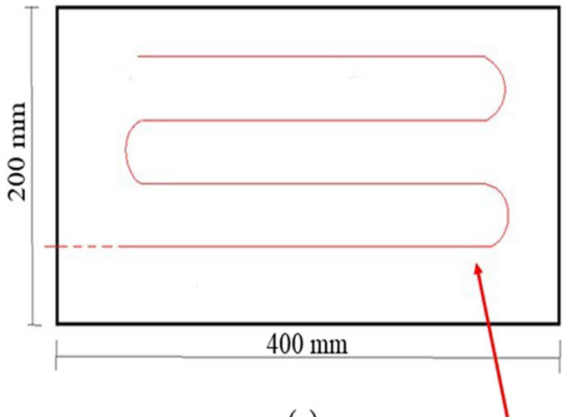

(a)

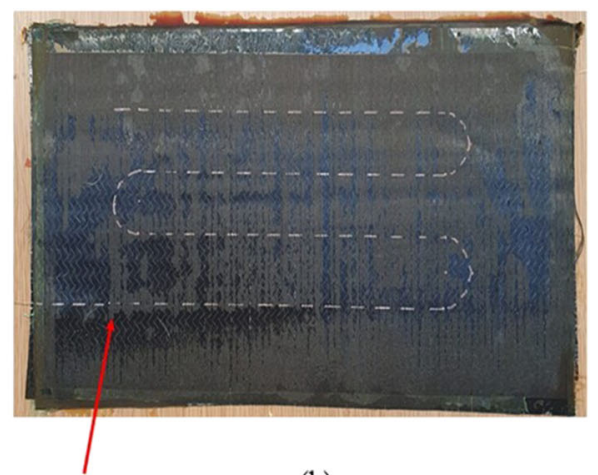

(b)

Path of embedded optical fibre sensor

Fig. 2 (a) Schematic of the composite plate and (b) photograph of the plate after manufacture

of the hole was increased, according to the details provided in Table 1. During each cycle of loading, distributed strain data was collected from the DOFS via an optical frequency domain reflectometry (OFDR) based interrogator from Luna Inc. (Roanoke, VA, USA). The selected hardware set-up enabled strains to be measured with a gauge length of $1.25 \mathrm{~mm}$ which allows to obtain several differently distributed strain readings.

Strains measured every $5 \mathrm{~mm}$ along the length of the optical fibre were selected from the large dataset collected. These selected strains were then used to initialise, train, and test a set of neural networks, in accordance with the procedure described in Section 2.

\section{Results and Discussion}

The results of the procedure's implementation are herein presented and discussed. Firstly, Figs. 3, 4 and 5 show the contour plots of the strains collected by the optical fibre sensor at the most severe loading condition (i.e. the maximum applied load) during loading cycles 1,9 , and 11 , respectively, for each of the represented points, that correspond to the selected strain measurement locations along the length of the optical fibre sensor (5 mm apart). The first loading cycle

Table 1 Four-point bending tests details - number, maximum achieved load and entity of drilled hole

\begin{tabular}{lll}
\hline Test number & Maximum load $(\mathrm{kN})$ & Hole diameter $(\mathrm{mm})$ \\
\hline 1 & 0.55 & No hole \\
2 & 0.55 & 2.5 \\
3 & 0.80 & 2.5 \\
4 & 0.64 & 2.5 \\
5 & 0.80 & 3.0 \\
6 & 1.00 & 3.0 \\
7 & 1.00 & 4.0 \\
8 & 1.00 & 4.0 \\
9 & 1.00 & 6.0 \\
10 & 1.00 & 8.0 \\
11 & 1.50 & 8.0 \\
\hline
\end{tabular}


represents the one applied on the pristine configuration. All of the displayed strain values are expressed in micro-strains $(\mu \varepsilon)$ and their entity is expressed through the colorbar. Since the first experiment (Fig. 3), the map of measured strains has highlighted that the top part of the plate shows higher strains and, so, lower stiffness than the rest of the sample. This is likely due to the fact that the plate experienced a non-uniform infusion during the manufacturing process leading to an uneven final thickness. A similar trend has been found out for the other two cases (Figs. 4 and 5), where the higher values of measured strains are related to higher applied loads. As the deformation behaviour of the top edge of the plate is already evident during loading cycle 1 and as the strain data measured during that cycle were used to train the ANNs, it is clear that their training was established taking this condition into account. In addition, from Figs. 3, 4 and 5, it may be deduced that strain mapping is a useful tool to easily visualise the strain distribution across a structure and the possible strain concentration. Taking a look at the maps of strains measured during loading cycles 9 (Fig. 4) and 11 (Fig. 5) and quickly comparing the maximum compressive strain for each of them with the maximum compressive strain measured during loading cycle 1 (Fig. 3), a difference of about $750 \mu \varepsilon$ and $1050 \mu \varepsilon$ may be noticed, respectively.

Concerning the ANNs used for the current work, they are single-layer networks belonging to the family of Feed-forward Perceptron Networks trained via the Levenberg-Marquardt backpropagation algorithm [31] implemented in Matlab's Neural Network Fitting Tool [32]. As described in Section 2, the Performance Index has been calculated through Eqs. (2) and (3). Then, it has been averaged on the total number of ANNs $(N)$ and is reported in Fig. 6 in which it is plotted against the number of loading steps used for the training $\left(L_{R_{t}}\right)$.Analysing the figure it appears that the average Performance Index (that is a quantification of the training error) becomes stable approximately around 100 loading steps

As mentioned in the previous sections, the procedure in object is based on the idea that the locations far from the damage are not affected by it and so, as the ANNs are trained on the reference structure, they will continue to work as expected. On the contrary, as near the damage the strain distribution will be influenced by the damage itself, the 'damaged' strains -

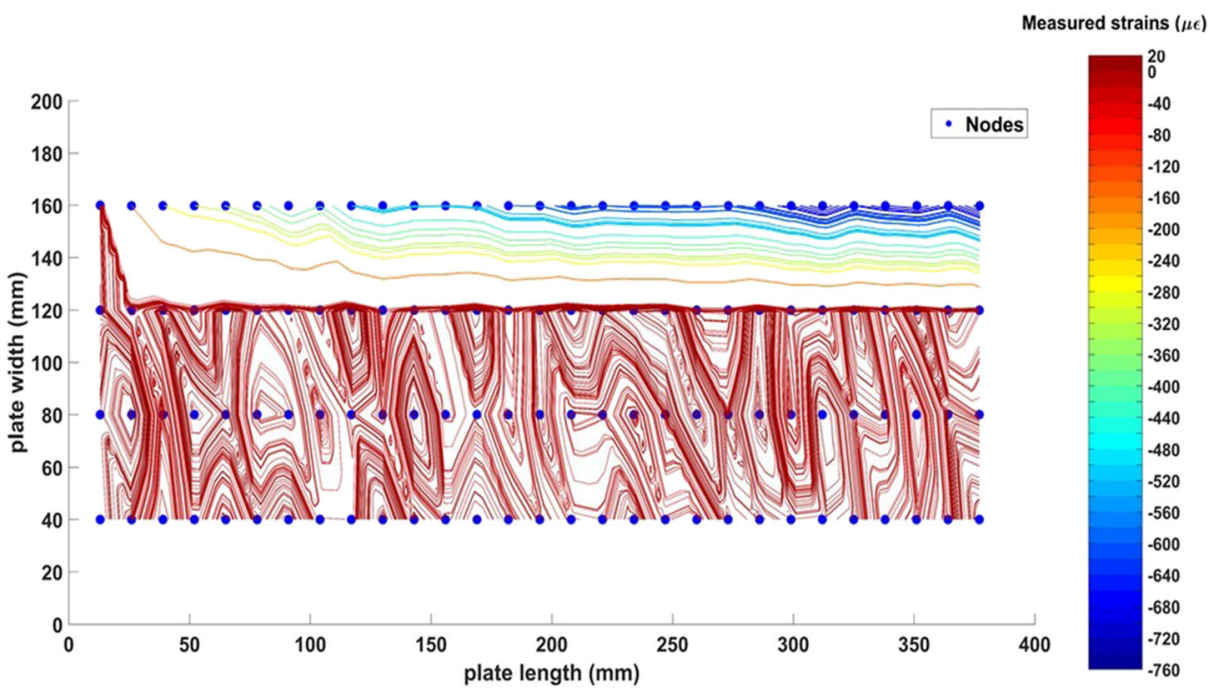

Fig. 3 Contour plot of the composite plate strains collected during Test $1($ max. applied load $=0.55 \mathrm{kN})$ 


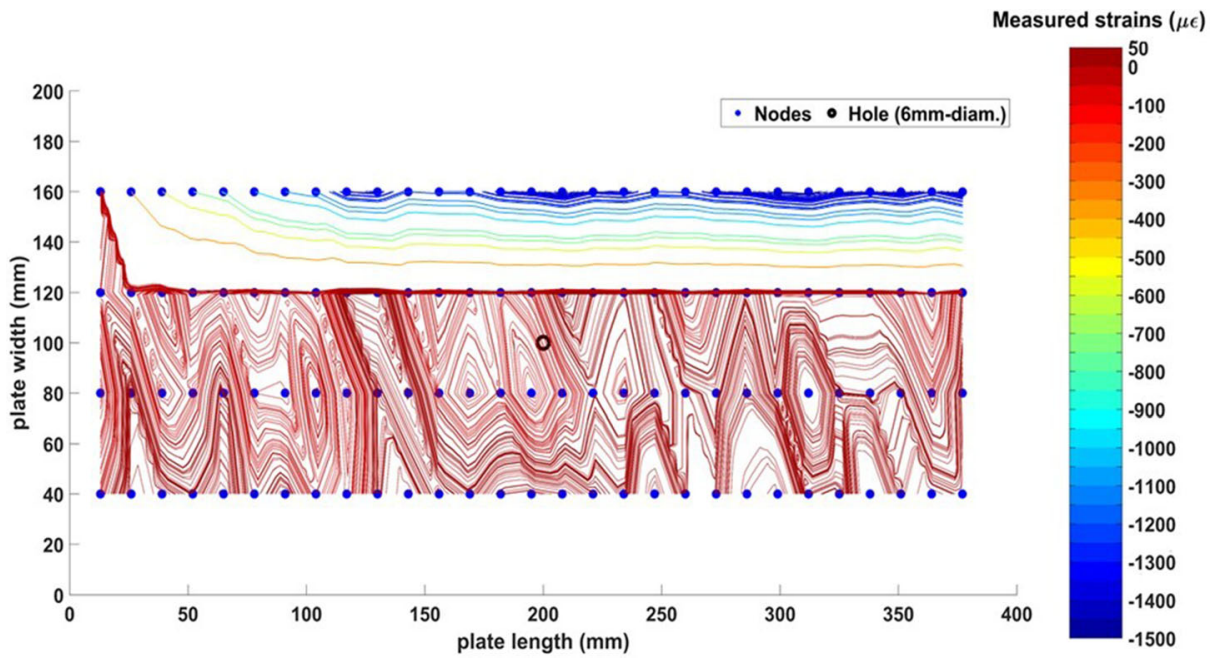

Fig. 4 Contour plot of the composite plate strains collected during Test 9 (max. applied load $=1.00 \mathrm{kN})$

that do not belong to the training set - provided to the ANNs will let the ANNs themselves produce 'out-of-scale' output data. This anomalous response received from the ANNs is reflected in high values for the quantity expressed in Eq. (1); in addition, it does not represent a quantification of the damage condition but only allows to understand that, where and when it happens, there is something to worry about or to simply take in consideration.

Figure 7 shows the results provided by the implemented procedure when applied on the same data used for the ANN training phase (i.e. strain data measured on the pristine plate configuration during loading cycle 1). The colour of the measurement points represents the entity of the mismatch evaluated by Eq. (1), expressed in $\mu \varepsilon$. The mismatch in Eq. (1) takes negligible values, as the data provided to the trained ANNs are the same that had been used during the training phase. This proves that the ANNs training was successful.

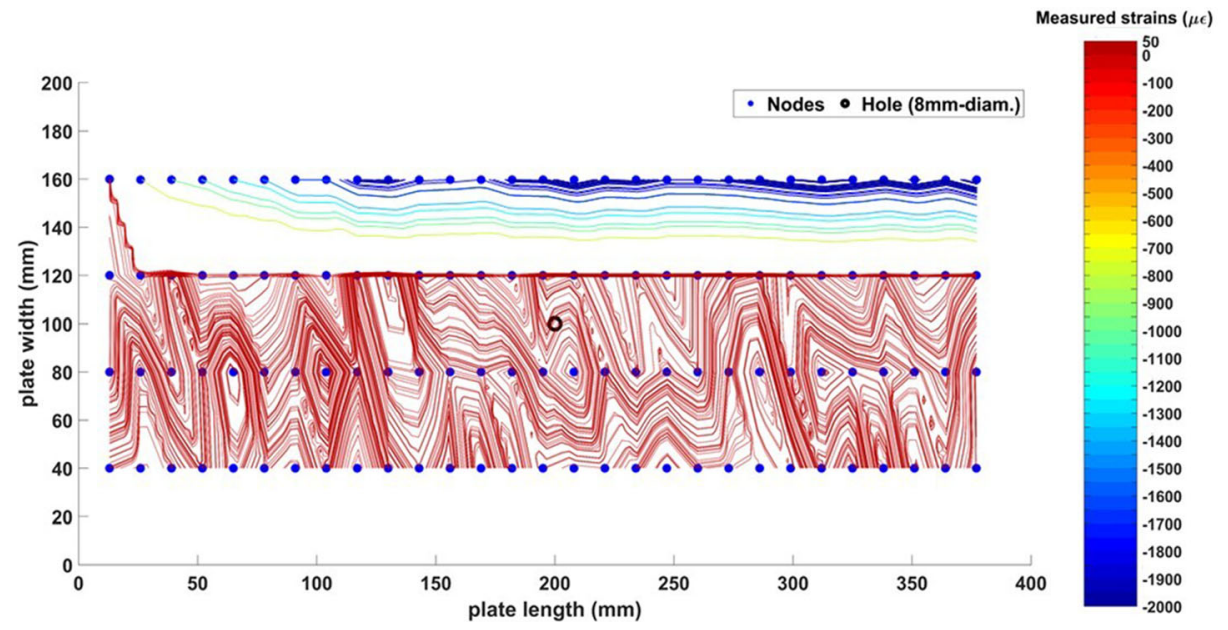

Fig. 5 Contour plot of the composite plate strains collected during Test 11 (max. applied load $=1.50 \mathrm{kN})$ 


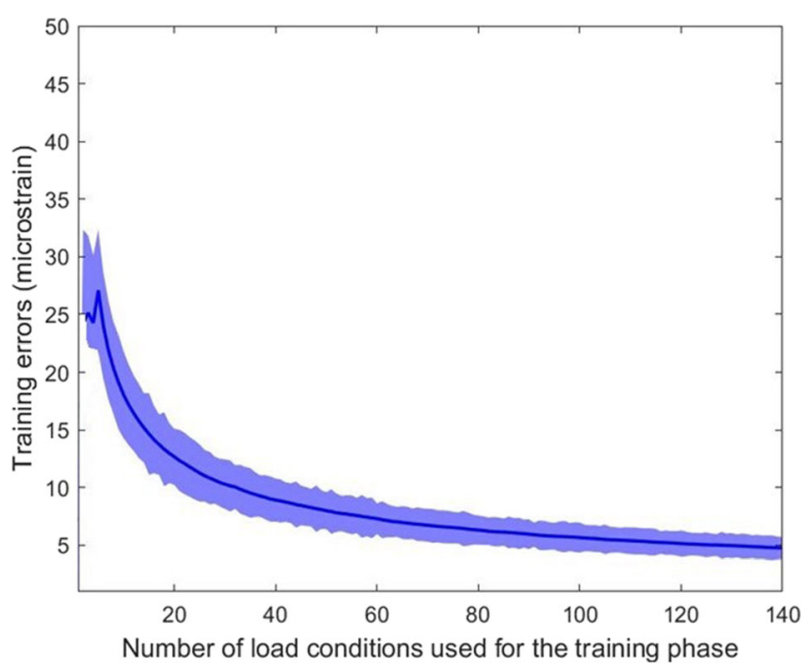

Fig. 6 Average training error of neural networks as function of training loading steps (blue line). The shaded region represents the confidence interval in terms of standard deviation

Figure 8 shows the results provided by the implemented procedure when applied on data measured on the 'damaged' configuration. The meaning of the represented graphical elements is the same explained for Fig. 8. In addition, in Fig. 7 there is the representation of the hole drilled in the plate (empty circle in the plate centre).

In particular, Fig. 8 shows the results of the ANN procedure for the 9th and 11th cycles of loading on the plate, when the diameter of the hole was $6 \mathrm{~mm}$ and $8 \mathrm{~mm}$, respectively. The maximum evaluated value of $e$ (Eq. 1) is of about $1100 \mu \varepsilon$ (Fig. 8.a) or higher (1200 $\mu \varepsilon$ in Fig. 8.b), as visible through the colour bar.

According to the procedure logic, higher values of the mismatch $e$ are expected in the region close to the hole. However, in the current work, the algorithm is not able to capture the hole. Instead, the optical fibre section close to the top edge of the plate is highlighted as having higher mismatch values.

Consequently, the fact that Fig. 8 shows high values of the mismatch evaluated in Eq. (1), in correspondence of the same plate edge highlighted previously, means that during the loading cycles the plate experienced such a deterioration that caused the change of strain relationships in that region, captured by the ANNs procedure as expected. So, as the evaluation of the mismatch in Eq. (1) gives a stronger 'warning' signal, as seen in Fig. 8, than the traditional strain difference, the procedure has shown to be more sensitive to the structural changes than the evaluation of strain maps.

The use of ANNs to establish correlation among strains is fundamental because it means determining a function that is representative of the mechanical behaviour of the structure itself under given boundary conditions. The benefit of using ANNs is the possibility to exploit their generalisation capability [28].

In the present case, this means that if the baseline structure, subjected to a given loading condition, is characterised by a certain strain-correlation function (found as a result of the ANNs training), this function will still be valid, for instance, even in case of a different

${ }_{1}$ Loading steps are defined as the selected measurement intervals during the specified cycle of loading, as a function of time. 


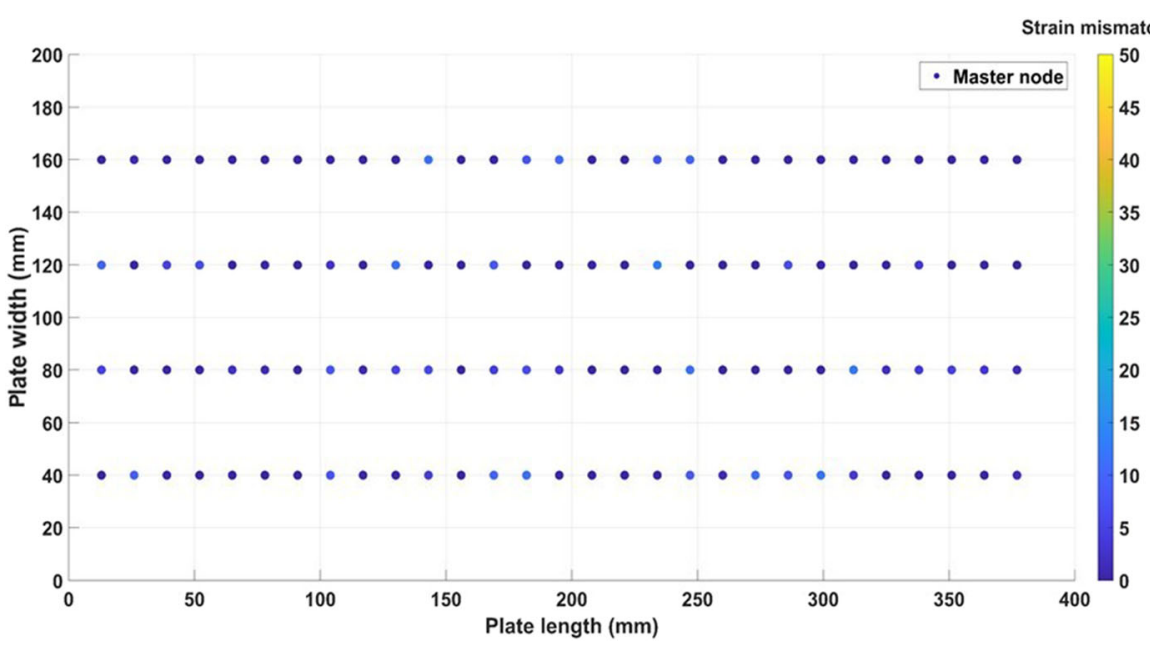

Fig. 7 Mismatch in strain correlations, as predicted by the ANN-based damage detection procedure, evaluated when the data provided to the ANNs are the same used for the training phase. The colour of each point represents the strain mismatch as per the scale bar to the right of the plot

maximum applied load - provided equal boundary conditions. Then, if the occurrence of damage caused a change in the mechanical features of the structure, the previous straincorrelation function would not be valid anymore, due to the change in the spatial dependence of strains from each other. So, the novelty of the proposed approach stands in the possibility to infer the eventual damage state of a structure evaluating the change of the aforementioned function, instead of simply looking at the strain mapping shape and values that could be insufficient for the intended purposes, as seen in the previous sections.

In addition, some of the studies highlighted in Section 1.3 have showed that strain field mappings are often related to stress-strain relationships calculation, damage patterns and/or data reduction operations. Therefore, measuring variation of strains correlation using neural networks appears to be easier, less time-consuming and more flexible than other strain-based methodologies.

This is due to the fact that there is no need to:

(i) associate damage patterns to strains. This means that data do not need to be reduced or characterised with computational-expensive procedures;

(ii) rely on stress-strain relationships. This means that the material properties are not necessary to implement the procedure and, therefore, it is not needed to calculate them analytically or numerically.

So, as this approach only relies on the evaluation of relationships between strains and how they could change due to the presence of damage, the procedure could be used for any kind of material, even for those whose properties are not known. In this way, the whole algorithm could be implemented on any kind of structure, even if it is a first-time seen one.

Finally, it must be noticed that the hole did not represent a significant damage configuration on the evaluated structure. This is mainly due to its small dimension and to its neatness that make it undetectable and, consequently, to the fact that the DOFS is not enough close to the hole itself. In terms of damage-tolerance and fatigue evaluation philosophy, as explained in Section 1, this damage belongs to the aforementioned 'Category 1 damage' [11]. An additional reason. 

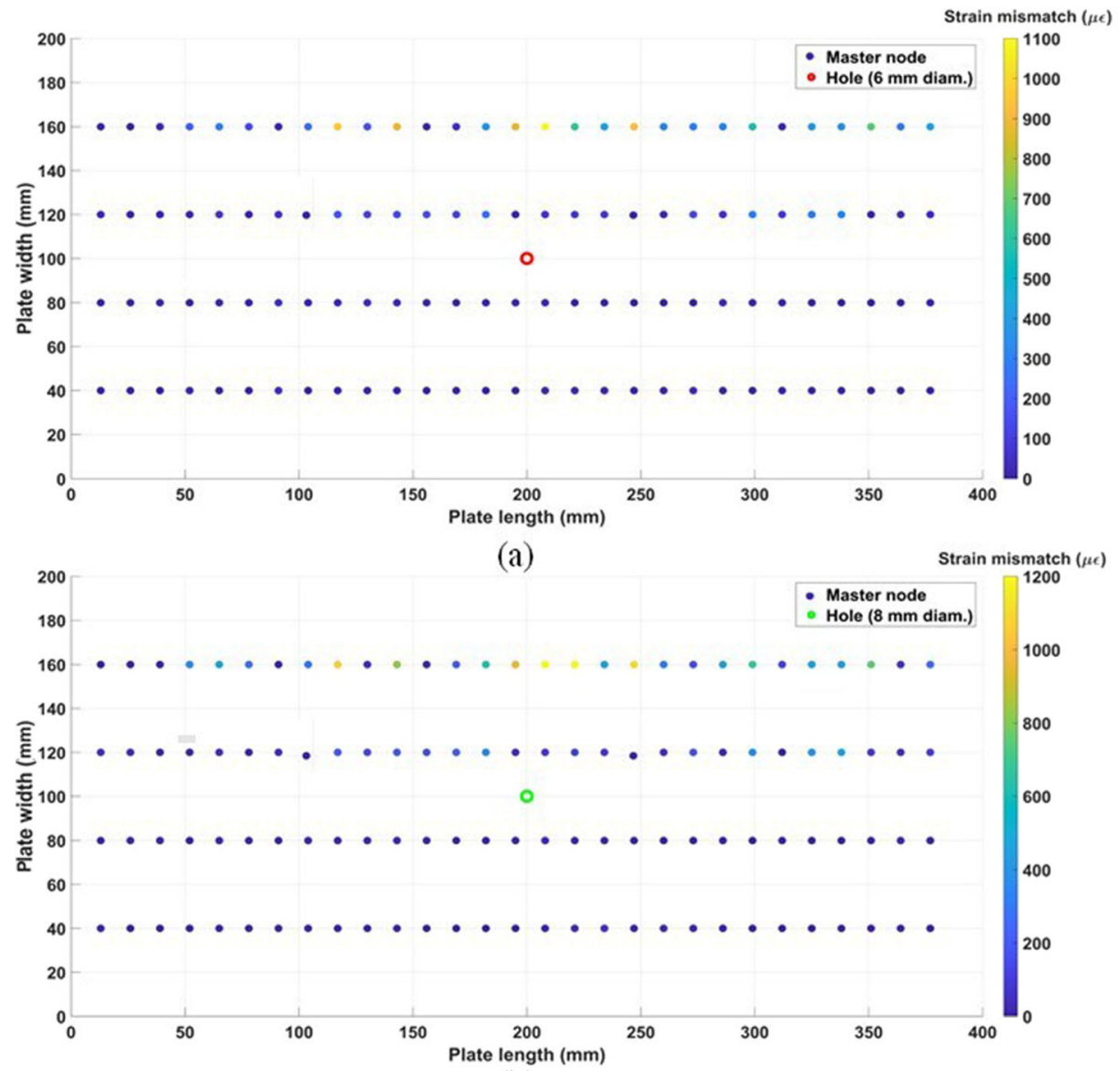

(b)

Fig. 8 Mismatch in strain correlations as predicted by the ANN-based damage detection procedure: (a) during loading cycle $9($ load $=1.00 \mathrm{kN})$, and $(\mathbf{b})$ during loading cycle $11(\mathrm{load}=1.50 \mathrm{kN})$

\section{Concluding Remarks}

The current work presents an implementation of a method for the structural health monitoring of composite structures supported by artificial neural networks. The algorithm is based on establishing correlations among strains measured in a given area of the structure under investigation. Any change in those correlations that may be caused by the presence of damage, propagation of defects and flaws, may lead to the detection of them. The algorithm was implemented on data collected by a distributed optical fibre sensor embedded in a composite plate subjected to several four-point bending tests.

The novelty of the proposed approach stands in its independence from both the loads applied on the structure and from the kind of material the structure is made of. In contrast with other machine-learning-based SHM techniques, the presented ANNs-based approach does not need to be supported by data reduction procedures and, as the algorithm is trained using only 'positive' (i.e. healthy) samples, it does not need to be associated to damage patterns. For these reasons, the proposed method is less time-consuming and less computational-expensive and, so, it may be 
useful in real-life structural health monitoring applications. In addition, the procedure has shown to be more sensitive to the change in strain relationships than the traditional strain maps; in particular, the change in strain relationships were about $200 \mu \varepsilon$ higher than strain variation evaluated through strain mappings. Finally, the algorithm was able to identify anomalous situations even if they were not significantly worrying under a structural point of view.

As the introduced damage falls into the undetectable damage categories, in near future work the sample should be subjected to more noticeable damage configurations, in order to better explore the structure's capabilities especially in case of more severe damage categories.

Acknowledgements This work is part of a collaboration with the Aerospace Research Institute of The University of Manchester that has been supporting the activities providing materials, equipment, know-how and excellent facilities.

Funding Information Open access funding provided by Università degli Studi della Campania Luigi Vanvitelli within the CRUI-CARE Agreement.

\section{Compliance with Ethical Standards}

Conflict of Interest The authors declared no potential conflicts of interest with respect to the research, authorship and/or publication of this article.

Open Access This article is licensed under a Creative Commons Attribution 4.0 International License, which permits use, sharing, adaptation, distribution and reproduction in any medium or format, as long as you give appropriate credit to the original author(s) and the source, provide a link to the Creative Commons licence, and indicate if changes were made. The images or other third party material in this article are included in the article's Creative Commons licence, unless indicated otherwise in a credit line to the material. If material is not included in the article's Creative Commons licence and your intended use is not permitted by statutory regulation or exceeds the permitted use, you will need to obtain permission directly from the copyright holder. To view a copy of this licence, visit http://creativecommons.org/licenses/by/4.0/.

\section{References}

1. Lukaszewicz, D.H.J.A., Ward, C., Potter, K.D.: The engineering aspects of automated prepreg layup: History, present and future. Compos. Part B Eng. 43, 997-1009 (2012). https://doi.org/10.1016/j. compositesb.2011.12.003

2. Califano, A., Grassia, L., D’Amore, A.: Fatigue of Composite Materials Subjected to Variable Loadings. J. Mater. Eng. Perform. 28, 6538-6543 (2019). https://doi.org/10.1007/s11665-019-04373-9

3. Perfetto, D., Lamanna, G., Manzo, M., Chiariello, A., di Caprio, F., di Palma, L.: Numerical and Experimental Investigation on the Energy Absorption Capability of a Full-Scale Composite Fuselage Section. Key Eng. Mater. 827, 19-24 (2019). https://doi.org/10.4028/www.scientific.net/kem.827.19

4. Diamanti, K., Soutis, C.: Structural health monitoring techniques for aircraft composite structures. Prog. Aerosp. Sci. 46, 342-352 (2010). https://doi.org/10.1016/j.paerosci.2010.05.001

5. Fritzen, C.P.: Vibration-based structural health monitoring - Concepts and applications. Key Eng. Mater. 293-294, 3-18 (2005). https://doi.org/10.4028/www.scientific.net/kem.293-294.3

6. De Luca, A., Perfetto, D., De Fenza, A., Petrone, G., Caputo, F.: Guided wave SHM system for damage detection in complex composite structure. Theor. Appl. Fract. Mech. 105, 102408 (2020). https://doi. org/10.1016/j.tafmec.2019.102408

7. Chandarana, N., Sanchez, D.M., Soutis, C., Gresil, M.: Early damage detection in composites during fabrication and mechanical testing. Materials (Basel). 10, (2017). https://doi.org/10.3390/ma10070685

8. Grassia, L., Iannone, M., Califano, A., D'Amore, A.: Strain based method for monitoring the health state of composite structures. Compos. Part B Eng. 176, 107253 (2019). https://doi.org/10.1016/j. compositesb.2019.107253

9. Bao, X., Chen, L.: Recent Progress in Distributed Fiber Optic Sensors. Sensors (Switzerland). 12, 86018639 (2012). https://doi.org/10.3390/s120708601 
10. Di Sante, R.: Fibre Optic Sensors for Structural Health Monitoring of Aircraft Composite Structures: Recent Advances and Applications. Sensors. 15, 18666-18713 (2015). https://doi.org/10.3390/s150818666

11. Irving, P.E., Soutis, C.: Polymer composites in the aerospace industry. Elsevier - Woodhead Publishing, Sawston (2013). https://doi.org/10.1016/C2013-0-16303-9

12. Cremona, C., Santos, J.: Structural health monitoring as a big-data problem. Struct. Eng. Int. 28, 243-254 (2018). https://doi.org/10.1080/10168664.2018.1461536

13. Tibaduiza, D., Torres-Arredondo, M., Vitola, J., Anaya, M., Pozo, F.: A damage classification approach for structural health monitoring using machine learning. complexity (2018). https://doi.org/10.1155/2018/5081283

14. Dervilis, N., Choi, M., Taylor, S.G., Barthorpe, R.J., Park, G., Farrar, C.R., Worden, K.: On damage diagnosis for a wind turbine blade using pattern recognition. J. Sound Vib. 333, 1833-1850 (2014). https://doi.org/10.1016/j.jsv.2013.11.015

15. Oh, B.K., Glisic, B., Kim, Y., Park, H.S.: Convolutional neural network-based wind-induced response estimation model for tall buildings. Comput. Civ. Infrastruct. Eng. 34, 843-858 (2019). https://doi. org/10.1111/mice. 12476

16. Ewald, V., Groves, R.M., Benedictus, R.: DeepSHM: a deep learning approach for structural health monitoring based on guided Lamb wave technique. 19 (2019). https://doi.org/10.1117/12.2506794

17. Alvarez-Montoya, J., Carvajal-Castrillón, A., Sierra-Pérez, J.: In-flight and wireless damage detection in a UAV composite wing using fiber optic sensors and strain field pattern recognition. Mech. Syst. Signal Process. 136, 106526 (2020). https://doi.org/10.1016/j.ymssp.2019.106526

18. Panopoulou, A., Roulias, D., Loutas, T.H., Kostopoulos, V.: Health monitoring of aerospace structures using fibre Bragg gratings combined with advanced signal processing and pattern recognition techniques. Strain. 48, 267-277 (2012). https://doi.org/10.1111/j.1475-1305.2011.00820.x

19. Japkowicz, N.: Concept-learning in the absence of counter-examples: an autoassociation-based approach to classification. Ijcai. 169 (1999)

20. Christian, W.J.R., DiazDelaO, F.A., Patterson, E.A.: Strain-based damage assessment for accurate residual strength prediction of impacted composite laminates. Compos. Struct. 184, 1215-1223 (2018). https://doi. org/10.1016/j.compstruct.2017.10.022

21. Sierra-Pérez, J., Güemes, A., Mujica, L.E.: Damage detection by using FBGs and strain field pattern recognition techniques. Smart Mater. Struct. 22, (2013). https://doi.org/10.1088/0964-1726/22/2/025011

22. Nag-Chowdhury, S., Bellégou, H., Pillin, I., Castro, M., Longrais, P., Feller, J.F.: Crossed investigation of damage in composites with embedded quantum resistive strain sensors (sQRS), acoustic emission (AE) and digital image correlation (DIC). Compos. Sci. Technol. 160, 79-85 (2018). https://doi.org/10.1016/j.compscitech.2018.03.023

23. Flament, C., Salvia, M., Berthel, B., Crosland, G.: Local strain and damage measurements on a composite with digital image correlation and acoustic emission. J. Compos. Mater. 50, 1989-1996 (2016). https://doi. org/10.1177/0021998315597993

24. Katsikeros, C.E., Labeas, G.N.: Development and validation of a strain-based Structural Health Monitoring system. Mech. Syst. Signal Process. 23, 372-383 (2009). https://doi.org/10.1016/j.ymssp.2008.03.006

25. Güemes, A., Fernández-López, A., Fernandez, P.: Damage detection in composite structures from fibre optic distributed strain measurements. In: 7th European Workshop on Structural Health Monitoring. pp. 528535., Nantes: (2014)

26. Dey, S., Mukhopadhyay, T., Adhikari, S.: Uncertainty quantification in laminated composites (2018)

27. Jollivet, T., Peyrac, C., Lefebvre, F.: Damage of composite materials. Procedia Eng. 66, 746-758 (2013). https://doi.org/10.1016/j.proeng.2013.12.128

28. Grossi, E., Buscema, M.: Introduction to artificial neural networks. Eur. J. Gastroenterol. Hepatol. 19, 10461054 (2007). https://doi.org/10.1097/MEG.0b013e3282f198a0

29. Goren, A., Atas, C.: Manufacturing of polymer matrix composites using vacuum assisted resin infusion molding. Arch. Mater. Sci. Eng. 34, 117-120 (2008)

30. International, A.S.T.M.: D7264/D7264M-15 Standard test method for flexural properties of polymer matrix composite materials (2015)

31. Lourakis, M.I., a: A brief description of the Levenberg-Marquardt Algorithm implemened by levmar. Matrix. 3, 2 (2005). https://doi.org/10.1016/j.ijinfomgt.2009.10.001

32. Beale, M.H., Hagan, M.T., Demuth, H.B.: Neural Network Toolbox TM User's Guide R2013b. (2013)

Publisher's Note Springer Nature remains neutral with regard to jurisdictional claims in published maps and institutional affiliations. 